\title{
POPULATION SYNTHESIS OF COMPOSITE SYSTEMS
}

\author{
Andrew Pickles \\ Kapteyn Laboratorium, \\ Postbus 800,9700 AV Groningen, \\ The Netherlands
}

\begin{abstract}
Metallicity and age dispersion among the stars comprising stellar composite systems are discussed, with emphasis on the present and future implications for the technique of population synthesis.
\end{abstract}

\section{INTRODUCTION}

In preparing this talk I have referred to a wealth of excellent material, too extensive to list here in full. In particular however, I have tried to avoid excessive repetition of topics already amply reviewed by Burstein (1985), O'Connell (1985) and Whitford (1985a,b).

The identification of two distinct populations by Baade (1944) laid the framework for the concept of discrete stellar populations within composite systems. In particular he identified M32 and the bulge of M31 as pure population II, analagous to the oldest and lowest abundance tracers in our Galaxy. This established the expectation that purely Stellar Composite Systems (SCS), with no gas or dust, would consist entirely of metal-weak and old stars formed in the earliest stages of galaxy formation.

The idea that stellar populations could be well represented by a few essentially discrete points within a four-fold continuum of age, metallicity, mass function and population dynamics implies a similar separability in star formation history. The initial expectation that spiral bulge and elliptical stars would prove to be metal-weak foundered early however with Morgan's (1956) observations of strong lines in the brighter systems, prompting an extensive reapraisal of stellar characteristics in these systems. Subsequent work established the morphological and photometric similarity between spiral bulges and ellipticals (de Vaucouleurs 1961, Johnson 1966a, Frogel et. al. 1978 FPAM). Faber (1977) emphasised their similarity of line strength gradients, and Whitford (1978) identified the Galactic bulge as being spectroscopically similar to other spiral bulges and ellipticals.

Early narrow-band and scanner work to describe the integrated light of galaxies in terms of their stellar constituents was instrumental in 
establishing the high abunidance of bright systems and the variation of mean metallicity with absolute magnitude (Wood 1966; McClure and van den Bergh 1968; Spinrad and Taylor 1971 - ST; Faber 1972, 1973). Faber (1977) summarised the evidence that mean metallicity decreasing with luminosity was the basis for the colour-magnitude relation observed in SCS (Visvanathan and Sandage 1977). Subsequent work confirmed this (FPAM; Aaronson et. a 1. 1978 - ACMM; Frogel, Persson and Cohen 1980).

At this point perhaps the safest interpretation of stellar populations in SCS was that they were of similar age (dynamics and mass function), but distinguished by their mean metallicity as a consequence of initially differing galaxy mass. By identifying the age as that of Galactic Globular Clusters (GGCs), this hypothesis has attraction as satisfying much of the observational data and being not too distant from Baade's original and powerful arguments.

There are problems with this picture however. It is a fact that no synthesis of an SCS has achieved a fit without at least one of : i) inclusion of a young component in addition to a basically old population (Gunn, Stryker and Tinsley 1981 - GST; Bertola et. al. 1986), ii) inclusion of a metal-weak component in addition to a basically metalrich population (ACMM), iii) adoption of an intermediate age (roughly half that of GGCs) for the dominant population (Wood 1966; McClure and van den Bergh 1968; ST; Faber 1972, 1973; O'Connell 1976, 1980; Williams 1976; Pritchett 1977; Pickles 1985b; Rose 1985).

A comparison of synthesis fits shows them to be more remarkable for their similarities than differences. The problems of non-uniqueness in the presence of observational errors have been noted by several of the above and Peck (1980). But it is clear that this work has defined the general distribution of stellar numbers and light contributions within SCS, has converged to a relatively small range of acceptable mass function (power law) slopes, and mapped out the trend of mean metallicity with absolute magnitude.

I would suggest that a major source of residual disagreement comes not from observations or problems with synthesis techniques, but from attempts to derive global galaxy population parameters which are too restrictive. In essence I think the synthesis work tells us that SCS populations are truly composite, and should be interpreted in terms of distributions of (at least) metallicity and age.

More direct evidence for lack of metal-age uniformity in SCS comes from $U$ and particularly uv observations of ellipticals (Norgaard-Nielson and Kjærgaard 1981; Oke, Bertola and Capaccioli 1981; Wu et. al. 1980). The excess flux below 1800 A requires either a young or metal-weak component in an otherwise old and metal-rich system (Nesci and Perola 1985). Also the work of the Lick group indicates marked differences between Galactic globular clusters, M31 globulars and elliptical nuclei in terms of $\mathrm{HB}$ and $\mathrm{CN}$ line stengths, which can not be explained solely in terms of metallicity variations (Burstein et. al. 1984; Burstein 1985). Finally there has been a crescendo of direct work on stellar populations in local group systems, finding metallicity and age spread in globular clusters in the Magellanic clouds (Gascoigne 1980; Searle, Wilkinson and Bagnuolo 1980; Rabin 1982), in globulars and the halo of M31 (Burstein et. al. 1984, Mould and Kristian 1986), and evidence for 
metallicity spread (Whitford and Rich 1983, Terndrup, Rich and Whitford 1984) and young populations (Wood and Bessell 1983) in the Galactic bulge. This direct work more than any other perhaps is redefining the acceptable range of stellar populations in SCS.

I summarise below the evidence for both metallicity and age spread within SCS, and outline prospects for an expanded synthesis scheme based on available observations and theoretical data.

\section{METALLICITY DISPERSION WITHIN SCS}

\section{1 Synthesis fits with metallicity dispersion}

Several empirical population synthesis fits have included a range of stellar metallicities in their flux libraries. The strongest lined stellar components are usually required to match the strong lined composite spectra of bright systems such as the nuclei of M31 or bright ellipticals. Indeed a failure to completely match strong lines in galaxies has been taken as evidence for incompleteness in the stellar libraries at the high metallicity end. Fainter ellipticals have line strengths which can be well matched by existing libraries, but still with a range of metallicities from those available. Thus empirical models for M32 by McClure and van den Bergh (1968), ST and O'Connell (1980) utilise stellar components covering more than 1 dex in [Fe/H], from integrated globular cluster light to strong lined giant components. In fits to ellipticals covering a range of luminosities 1 also found most required a combination of different metallicity components (Pickles 1985b).

\subsection{Direct evidence for netallicity dispersion}

The most direct evidence for large metallicity spread within SCS comes from observations of stars in the Galactic centre and other local group systems. Whitford and Rich ( 1983 - WR) found a metallicity spread among Galactic bulge giants of more than 2 dex in [Fe/H]. Mould, Kristian and Da Costa (1983, 1984) found relatively small metallicity dispersions of 0.3 dex in NGC147 and 0.5 dex in NGC205 (dwarf elliptical companions to M31). Mould and Kristian (1986) find a dispersion of 1 dex or more in the halo of M31 however, and discuss the possibility of dispersion increasing with mean abundance.

\subsection{Metallicity dispersion in evolutionary syntheses}

Tinsley (1972) discussed the chemical enrichment process within ellipticals, and noted that steady enrichment during initial collapse and star formation could explain radial colour and and composition gradients. In general however, models by Tinsley and co-workers and by Bruzual (1983) have adopted a simpler scenario in which rapid star formation in the initial collapse produces a stellar population of roughly uniform composition, generally taken as solar for convenient 
application to existing isochrone and stellar data.

Arimoto and Yoshii (1986 - AY) have recently extended this work to explicitely allow for chemical enrichment during star formation within ellipticals. The effects included in their scheme are:

i) star formation rate (SFR) linearly proportional to the gas fraction until the thermal energy of the gas exceeds its binding energy, after which remaining (enriched) gas is expelled by a supernova driven galactic wind.

ii) metal enrichment based on star formation, nucleosynthesis and re-ejection via supernovae; numerical integration of the gas fraction and chemical enrichment equations occurs in time steps of $5.10^{\circ}$ years.

iii) evaluation of star formation at each time (and gas metallicity) step, for a range of SFR and initial mass function (IMF) parameters.

iv) evolution of all stars along the appropriate (Sweigart and Gross 1978) tracks.

By explicitely including chemical enrichment during star formation, AY $f$ ind that both the SFR and the IMF strongly affect the colours of galaxies. Metallicity enrichment is amplified and accelerated by having a giant dominated mass function, causing colour evolution (as well as luminosity evolution) to depend strongly on the IMF. These results can be compared with previous results (Tinsley 1972, Tinsley and Gunn 1976) which showed relatively small colour dependence on the IMF slope, but used single (solar) metallicity models.

The final (present day) stellar metallicity distribution derived by AY covers more than 2 orders of magnitude in [Fe/H]. This theoretically derived metallicity spread compares well with the distribution observed in the Galactic centre. I think the degree of correspondence with that observed by WR in a real SCS is both remarkable and gratifying. It is also very cautionary to all practitioners of the population synthesis technique.

AY also derive the variation of mean metallicity with total galactic mass. The variation with mass occurs because the supernovae driven thermal energy exceeds the gravitational binding energy earlier in lower mass galaxies, causing earlier (and proportionately larger) gas expulsion, and earlier termination of star formation.

\section{DWARF TO GIANT RATIOS AND M/L IN SCS}

A notable success of population synthesis has been the ability to determine mass to light ratios for the visible component to within quite high precision (factor of 2 roughly). Some early results gave mass to light ratios significantly higher than those determined dynamically for individual galaxies. These fits are now thought to be wrong for a combination of reasons; using too many late-type dwarfs to $f$ it the strong NaI lines, and putting too much reliance on early infrared data. Later work showed much lower values were preferable if proper account was taken of the cool giant population (O'Connell 1976). 
Since the lower main sequence dwarfs contribute so much mass and so little light, it is difficult to determine their contribution from medium resolution optical spectra. Detailed work on individual line strengths such as the FeH band at 990nm (Whitford 1977), and infrared features such as the $\mathrm{CO}$ band at $2.3 \mu$ and the $\mathrm{H}_{2} \mathrm{O}$ band at $1.9 \mu$ (FPAM; ACMM; Aaronson, Frogel and Persson 1978) show the presence of late giants, cooler and brighter than found in GGCs, and indicating $M / L$ values $\leq 10$. This corresponds to a (power law) IMF slope less than or equal to the commonly used Salpeter (1955) value of $x=1.35$. Evolutionary syntheses based on $f$ its to wide colour ranges have also converged to similar values (GST, $A Y$ ). It is important to note that population synthesis is really fitting the dwarf:giant ratio, particularly in the infrared, and mass functions other than a simple power law can also fit.

One important aspect of this is that population synthesis, unlike dynamical determinations, gives $M / L$ independent of distance. This has been a major impetus behind attempts to determine these values more precisely, by synthesising higher resolution near infrared spectra (Carter, Visvanathan and Pickles 1986) or CVF spectra of the CO band (Arnaud and Gilmore 1986, and this conference).

\section{AGE DISPERSION WITHIN SCS}

The most serious problem facing population synthesis is that of age determination of the stars forming the composite system. This is also the area in which there is most disagreement, real and percieved, between different approaches and between empirical as opposed to evolutionary synthesis. I would like to suggest that this is (in order of priority):

i) primarily due to external problems, particularly regarding isochrone placement in the HR diagram,

ii) partly due to interpretational problems, especially with regard to the tendency to summarise metallicity and age distributions in terms of single characterising parameters,

iii) partly due to real differences in synthesis techniques, specifically with respect to the relative weight placed on fitting continuum and spectral features, and

iv) only slightly due to problems with non-uniqueness, providing that the data quality is good $(S / N \geq 25)$.

\subsection{Isochrone placement}

Figure 1 shows two interpolated Yale isochrones (solid lines) I have used for evolutionary synthesis. The parameters are $(Z=0.017$, $Y=0.23, T=10 \mathrm{Gyr}, \quad \alpha=1.6)$. The metallicity changes were accomplished by direct interpolation among the Yale isochrones ( $\mathrm{Ciardullo}$ and Demarque 1977 - CD), and the ratio of mixing length to pressure scale height adjusted empirically (from $\alpha=1.0$ ) to the more popular value by applying Twarog's (1980) algorithm - that is by increasing Log T. by between 0.005 on the main sequence and 0.025 on the giant branch, without adjusting $M_{b o l}$. The amplitudes of these shifts are indicated by arrows. 


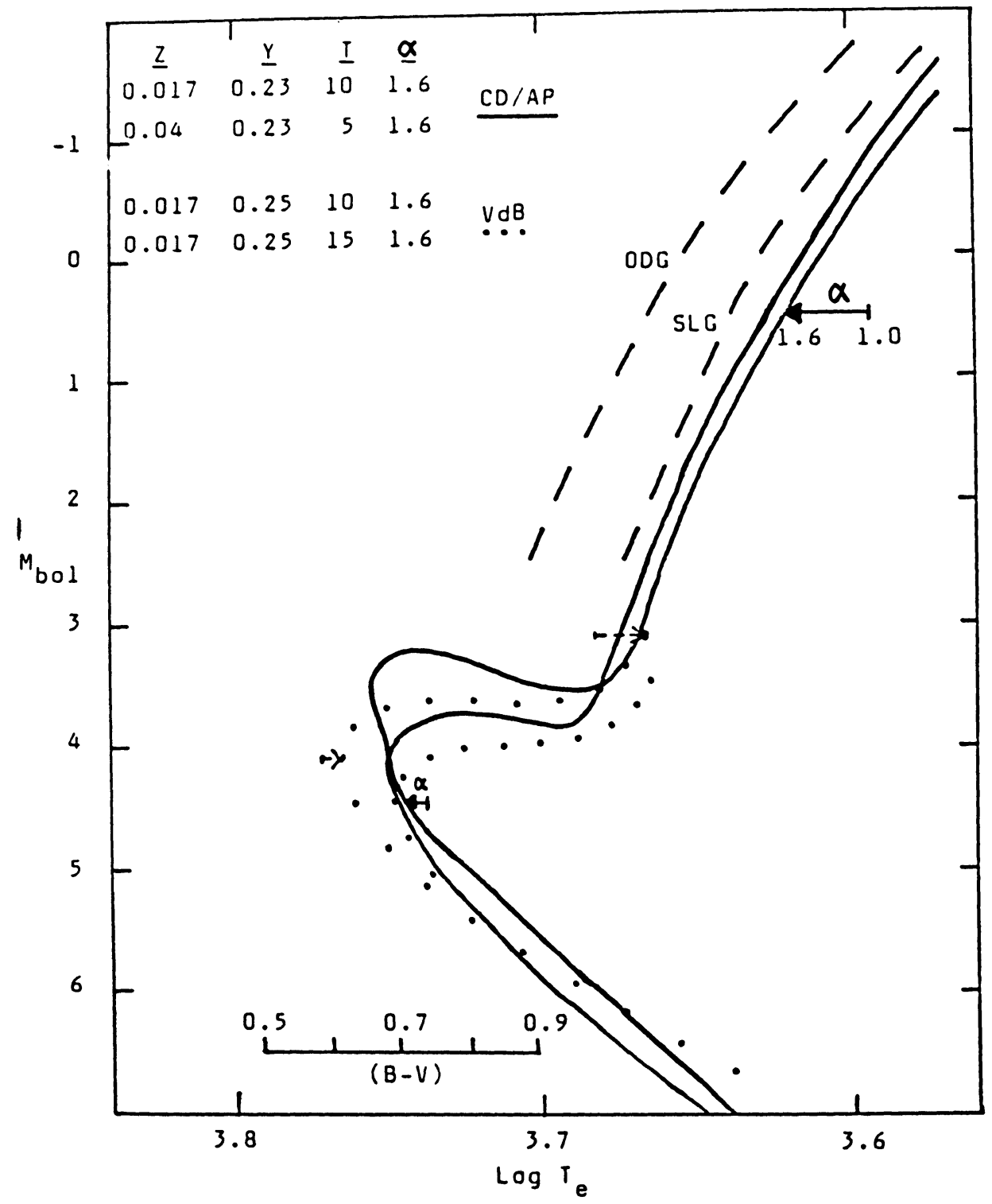

Figure 1: compares the position in the Mbol-LogTe plane of two interpolated and adjusted Yale isochrones (CD/AP, solid lines), and two shifted VandenBerg isochrones (VdB, dotted lines): The abundance, age and mixing length ratio parameters are given in the top LH corner. Arrows on the giant branch and main sequence indicate the empirical blueward shift applied to the rale isochrones to achieve the higher mixing length ratio, and the redward colour shift applied to the VandenBerg isochrones. Also shown are the positions (dashed lines) of the Old Disk Giant and Strong Lined Giant branches used by Gunn, Stryker and Iinsley. 
These isochrones are very close to the revised Yale isochrones (Green, Demarque and King 1985). At the bottom is a (B-V) colour scale from Johnson (1966b).

This diagram illustrates one problem concerning age dating via population synthesis. Empirical syntheses are not heavily constrained by a priori isochrone data, and typically give elliptical turnoff colours of (B-V) 0.7. This implies Yale ages of about $10 \mathrm{Gyr}$ for a solar metallicity population, or younger for a metal-rich population. In evolutionary syntheses of Virgo and Coma ellipticals, GST preferred redder turnoff colours of (B-V) 0.8, which clearly imply older ages. 0 'Connell (1985) has pointed out one major difference between the two approaches which concerns giant branch placement. Dashed lines in the top part of figure 1 indicate the giant branches used by GST. Both the old Disk Giant branch (ODG - used for GST's solar metallicity models) and the Strong Lined Giant branch (SLG - used for their metal-rich models) are substantially bluer than the Yale giant branches. This difference then results in a redder turnoff colour to compensate.

Clearly the question of isochrone placement is external to that of population synthesis. If we knew their correct placement this source of disagreement would disappear. Population synthesis is not a lottery; you can't get any answer you like, but you can get a range of answers depending on the framework in which you work - and this framework is not well defined at the higher metallicity end.

VandenBerg (1985) has recently calculated a new set of isochrones which explicitely allow for a higher mixing length ratio, aswell as using the latest opacity and nuclear reaction rate data; two of these are shown as dotted 1 ines in $f$ igure 1 . They have parameters $(Z=0.0169$, $Y=0.25, T=10$ and $15 \mathrm{Gyr}, \alpha=1.6)$, corresponding to solar metallicity. They have been shifted redward in colour according to the recipe given in VandenBerg (1985); this empirical shift is necessary to give reasonable fits to the sun with a $5 \mathrm{Gyr}$ solar metallicity isochrone, and to GGCs with lower metallicity isochrones.

The shifted VandenBerg isochrones are bluer than the corresponding Yale isochrones conly partially due to the small helium abundance difference), implying older ages for a given turnoff colour. From the point of view of population synthesis there is a very rough correspondence in turnoff colour (at B-V $\sim 0.7$ ) between a) a Yale isochrone of $2 w_{2}$ times solar metallicity and age 5 Gyr, b) a Yale isochrone of solar metallicity and age $10 \mathrm{Gyr}$, and c) a VandenBerg isochrone of solar metallicity and age $15 \mathrm{Gyr}$. VandenBerg (and others) are continuing to work on metal-rich isochrones, and it seems clear that population synthesis should play as significant a role here in $f$ itting theory to observations, as do direct observations of CM diagrams at lower metallicities. The point being that there are very few metal-rich populations accessible to direct CMD work.

\subsection{Effects of metallicity dispersion}

In view of the large metallicity dispersion present in galaxies, it is obviously pertinent to ask whether the blue turnoff colours indicated by empirical syntheses could be due to a large metal-weak component. 
Rose (1985) has dealt with this problem quite extensively for the particular case of M32. He $f$ inds that a metal-poor contribution of $8 \%$ light at $4000 \AA$ provides a good $\mathrm{fit}$ to his $\left(\mathrm{H}_{E}+\mathrm{Call} \mathrm{H}\right) / \mathrm{Call} \mathrm{K}$ index, whereas a much larger contribution of $20 \%$ would be needed to $f$ it the colours if all stars were old. Notably a metal-weak fraction of ${ }^{2} 8 \%$ is commensurate with that predicted by $A Y$. In fact it is also that predicted by Pagel and Patchett (1975) for approximately the same choice of heavy yield parameter which reproduces the WR results in the Galactic centre (Pagel 1985; Rich 1986).

In comparing observations with theory it is certainly better at this stage to look at galaxies of solar mean abundance or less. Existing stellar libraries are then more complete, and comparison with both Yale and VandenBerg isochrones is possible. Figure 2 shows the situation pertaining to three Fornax ellipticals (NGC1336, JJ51, JJ79) of roughly solar mean abundance, which have been synthesised with a flux library containing a range of metallicities (Pickles $1985 \mathrm{a}, \mathrm{b}$ ). Note that the temperatures of the stellar groups are fixed by their observed colours, but that the absolute magnitudes can be adjusted slightly, particularly on the giant branch. Only solar metallicity dwarfs and subgiants were available for these fits, but the flux library did contain horizontal branch stars (spectral types $B$ to $F$ ) and the fit adopts a $5 \% \mathrm{~V}$ light contribution from these stellar groups. The adjusted Yale isochrones shown (solid lines) are all of old age ( $15 \mathrm{Gyr}, \alpha=1.6$ ), and are of low, solar and high metal abundance. Notable features of figure 2 are:

i) The giant branch can be fitted by a purely old (15 Gyr) population of solar mean abundance and some metallicity dispersion. The age resolution is poor on the giant branch however, and the same can be said for younger ( $10 \mathrm{Gyr}$ or less) isochrones there.

ii) The main sequence requires a substantial contribution from blue stars. The numbers indicate that these would have to be all metalweak to fit a Yale $15 \mathrm{Gyr}$ isochrone. Younger isochrones provide a better fit to the main sequence and giant branch simultaneously.

iii) There is no compelling evidence from these (or other) synthesis fits to normal ellipticals for a substantial spread of ages. Most fits work well with a single (intermediate) age and a spread of metallicity.

What could be wrong here and is there any reasonable way to get a basically old population?

i) I could force in metal-weak giants and redden the turnoff colour slightly. This is not appropriate within the context of either the Yale or VandenBerg isochrones however.

ii) I could boost the horizontal branch contribution. But Rose (1985) finds an upper limit of $4 \%$ at $4000 \mathrm{~A}$ for these hot stars in both field and Virgo ellipticals, by fitting to the He/Call ratio. The horizontal branch fraction here contributes $5 \%$ at $\mathbf{V}$ or about $8 \%$ at 4000A, so is already excessive.

iii) Rose's restrictions on hot stars apply to blue stragglers or uv bright stars aswell, so I cannot add these stars freely to provide the blue light. 


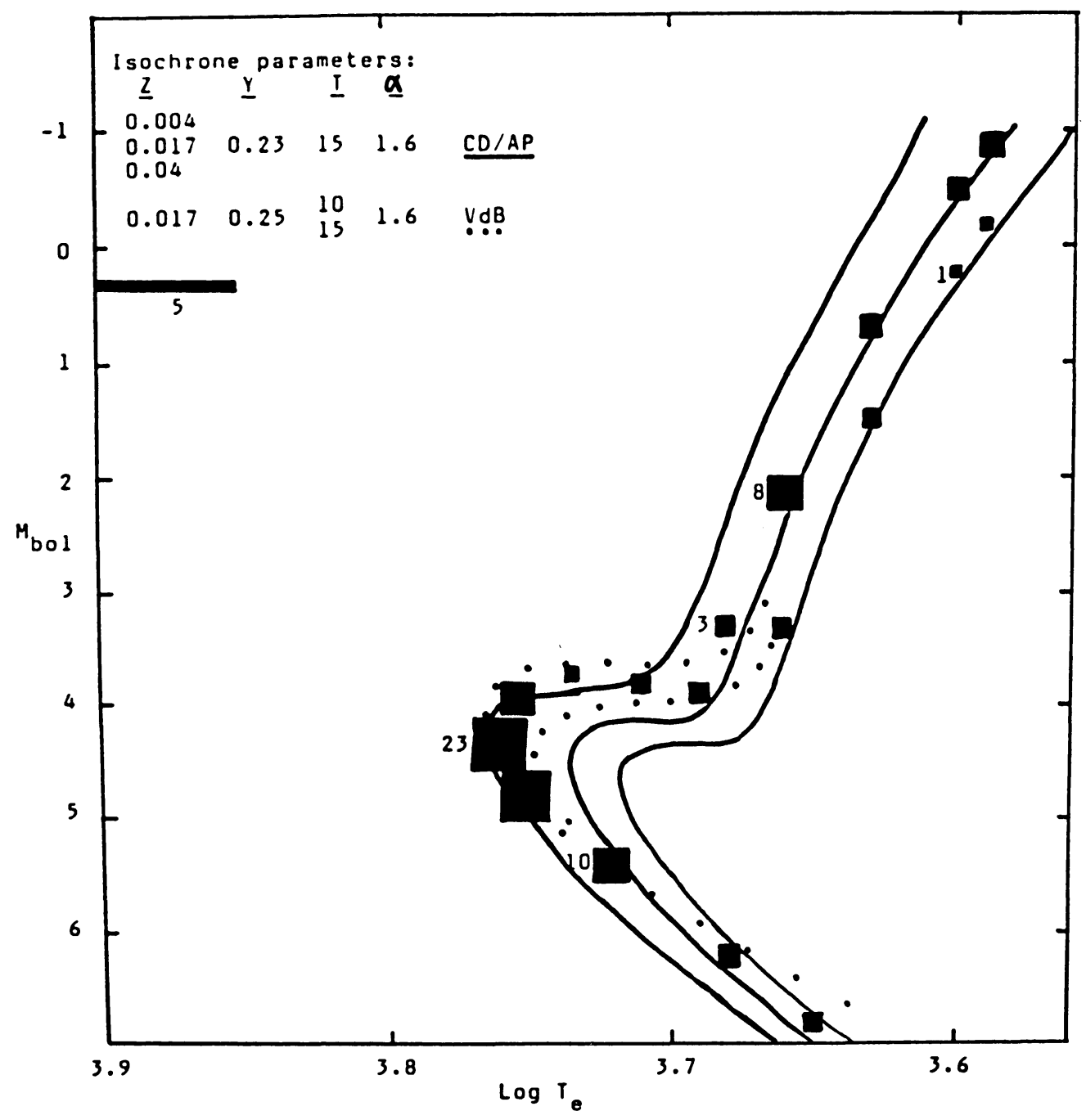

Figure 2: The mean synthesised light distribution of 3 Fornax ellipticals (solar mean abundance) is represented by discrete squares. Each square represents the location in the Mbol-LogTe plane of a stellar group used in the synthesis; their area is proportional to the percentage $V$ light contributed by that group, with numbers given for representative groups. The horizontal branch contribution is represented by a rectangle extended in temperature.

The synthesised light distribution is compared with 3 interpolated and adjusted Yale isochrones (solid lines) of weak solar and rich metal abundance, all of age 15 Gyr. Also shown are Vandenberg's 10 and 15 Gyr, solar metallicity isochrones (dotted lines) from figure 1 . 
Use of the VandenBerg isochrones (dotted lines) clearly gives ages older than indicated by the Yale isochrones, but the light distribution is still not well fitted by purely old isochrones. The presence of a blue turnoff appears to be unavoidable within the context of either Yale or VandenBerg isochrones; this is strongly supported by Rose's higher resolution data which sets strong limits on the permissible contributions from hot stars which could otherwise compensate for a redder turnoff. A blue turnof $f$ is even more pronounced for ellipticals like M32 (O'Connell 1980, 1985; Rose 1985). The prospects of satisfactorily fitting all or even some ellipticals with purely old isochrones just do not look good, even allowing for wide metallicity dispersion. Intermediate ages, or at least a significant age dispersion, appear to be a necessary component of these 'simple' systems.

\subsection{Origin of age dispersion}

Bruzual (1983) has calculated models of elliptical galaxy light distribution as a function of lookback time, which have been extensively used in the analysis of galaxy colours in clusters of high redshift. Bruzual's (solar metallicity) models are characterised by a parameter ' $\mu$ ' which describes the exponential rate at which stars form out of the original gas. Without getting too deeply into this additional but related topic, it seems clear that observations of galaxies at large distances are mostly contained within the envelope specified by $\mu=1.0$ (effectively instantaneous star formation) and $\mu=0.5$ (half the gas converted to stars in $1 \mathrm{Gyr}$ ) - (Lilly and Longair 1984; Eisenhardt and Lebof sky 1986).

It is interesting to compare Bruzual's models, which are quite well known, with those of Arimoto and Yoshii, which are less so. The $A Y$ models use a parameter ' $v$ ', which quantifies the SFR (per unit mass of residual gas) in units of the PDSFR in the solar neighborhood. Despite the fact that gas conversion to stars is not exactly an exponential in the AY models (and they explicitely reject the instantaneous recycling approximation), there is a rough analogy which is given here (Arimoto 1986 - private communication):

Arimoto and Yoshii $v$

$\begin{array}{rl}1 & 0.1 \\ 6 & 0.5 \\ 11 & 0.7 \\ 21 & 0.9 \\ 42 & 0.99\end{array}$

The star formation rate determined by AY to best fit present day giant elliptical colours, assuming star formation started 15 Gyr ago, is $v=42$, corresponding to rapid star formation and a galactic wind occurring less than 1 Gyr after its onset. Their star formation rate parameter would decrease however if a higher mass function slope were used (they used $x=0.95$ in Tinsley's notation), and particularly if they 
adjust their models to a higher value of the mixing length ratio. They have used stellar colours derived from Sweigart and Gross (1978) evolutionary tracks for a mixing length ratio of $\alpha=1.0$; increasing this makes the component stars bluer, necessitating a slower SFR to match present day galaxy colours. An estimate of the SFR parameter appropriate to a mixing length ratio of $\alpha=1.6$ is $v=10$ - 20 (Arimoto 1986, private communication), implying quite extended star formation terminating after 5-10 Gyr with the onset of a galactic wind. These calculations are being repeated for the higher ratio of mixing length to pressure scale height.

\section{CONCLUDING REMARKS}

It may be possible to reconcile all the observations and 'classical' concepts of elliptical galaxy formation with temporally extended star formation, culminating in a galactic wind which ejects hot, metal-enriched gas into the intergalactic medium. It seems to be possible to reproduce the colours this way; it should also be possible to construct synthetic spectra appropriate to the AY models for comparison with observations.

If an extended but declining star formation process cannot reproduce the observed spectra, then another source of gas for intermediate age star formation must be found. This could come from mergers, which qualitatively at least might explain the radial variation in clusters of the Elliptical to Spiral ratio. As a stochastic process they might also explain the colour range amongst dominant galaxies in clusters at large lookbacks. An alternative source of gas for late star formation might be infall of ejected gas, which might occur preferentially onto galaxies in the centre of the cluster potential well.

The key to unravelling these uncertainties possibly lies in a study of the radial variation of stellar populations in nearby ellipticals. This is necessary to enable quantitative discussion of population variations following dynamical interactions such as mergers or cooling flows. It is also necessary to rationally compare distant (whole) galaxies with nearby elliptical nuclei. Existing data indicate that radial variations are more complicated than a simple radial decline in mean abundance (Efstathiou; Peletier, Valentijn and Jameson; this conference).

Good spectra of distant galaxies at large lookbacks have been and can be obtained. These should provide a reliable check on the time evolution of stellar populations within galaxies. For synthesis however, it is necessary that the data be of sufficient quality to enable good deconvolutions.

Finally, since population synthesis is only a poor substitute for direct CMD work (but all that is possible outside the local group), every effort should be made to feed the expanding knowledge on local group populations into the synthesis process.

I thank the organisers and participants for a pleasant and memorable conference. 


\section{REFERENCES}

Aaronson M, Cohen J.G, Mould J \& Malkan M, (ACMM), 1978, Ap. J. 223, 824 Aaronson M, Frogel J.A. \& Persson S.E, 1978, Ap. J. 220, 442

Arimoto N. \& Yoshii Y, (AY), 1986, $A s t r$. Ap. (in press)

Arnaud K.A. \& Gilmore G, 1986, HNRAS, 220, 759

Baade W, 1944, Ap. J. 100, 137

Bertola F, Gregg M.D, Gunn J.E. \& Demler A, 1986, Ap. J. 303, 624

Bruzual A.G, 1983, Ap. J. 273, 105

Burstein D, 1985, PASP, 97, 89

Burstein D, Faber S.M, Gaskell C.M. \& Krumm N, 1984, Ap. J. 287, 586

Carter D, Visvanathan N. \& Pickles A.J, 1986, preprint

Ciardullo R.B. \& Demarque P, (CD), 1977, Trans.Astr.0bs, Yale Univ, v33

Eisenhardt P.R.M. \& Lebofsky M.J, 1986, Ap. J. (in press)

Faber S.M, 1972, Astr. Ap. 20, 361

1973, Ap. J. 179, 731

1977, in The evolution of Galaxies and Stellar Populations,

B.M. Tinsley \& R.B. Larson, eds. (New Haven, Yale Univ. Obs.), p177

Frogel J.A, Persson S.E, Aaronson M. \& Matthews K, (FPAM), 1978, Ap. J. 220, 75

Frogel J.A, Persson S.E. \& Cohen J.G, 1980, Ap. J. 240, 785

Gascoigne S.C.B, 1980, in IAU symposium 85, Star Clusters, ed. J.E. Hesser, Dordrecht, Reidel, p305.

Green E.M, Demarque P. \& King C.R, 1985, BAAS, 16, 51.06

Gunn J.E, Stryker L.L. \& Tinsley B.M, (GST), 1981, Ap. J. 249, 48

Johnson H.L, 1966a, Ap. J. 143, 187

1966b, Ann. Rev. Astr. Ap. 4, 193

Lilly S.J. \& Longair M.S, 1984, HNRAS, 211, 833

Morgan W.W, 1956, PASP, 68, 509

Mould J. \& Kristian J, 1986, preprint

Mould J.R, Kristian J. \& Da Costa G, 1983, Ap. J. 270, 471

1984, Ap. J. 278, 575

McClure R.D. \& van den Bergh S, 1968, Astron. J., 73, 313

Nesci R. \& Perola G.C, 1985, Astr. Ap. 145, 296

Nørgaard-Nielsen H.U. \& Kjærgaard P, Astr. Ap. 93, 290

O'Connell R.W, 1976, Ap. J. 206, 370

$1980, A p . J .236,430$

1985, in Spectral Erolution of Galaxies, C. Chiosi \& A.

Renzini, eds. (Dordrecht, Reidel), p321

Oke J.B, Bertola F. \& Capaccioli M, Ap. J. 243, 453

Pagel B.E.J, 1985, in symposium on Cosmogonical-Processes, Boulder, Colorado

Pagel B.E.J \& Patchett B.E, 1975, INRAS, 172, 13

Peck M.L, 1980, Ap. J. 238, 79

Pickles A.J, 1985a, Ap. J. Supp 1, 59, 33

1985b, Ap. J. 296, 340

Pritchett C, 1977, Ap. J. SuppI, 33, 397

Rabin D, 1982, Ap. J. 261, 85

Rich R.M, 1986, Conf. on Stellar Populations, Baltimore, USA

Rose J.A, 1985, Astron. J. 90, 927 
Salpeter E.E, 1955, Ap. J. 121, 161

Spinrad H. \& Taylor B.J. (ST), 1971, Ap. J. Supp1, 22, 445

Sweigart A.V. \& Gross P.G, Ap. J. SuppI, 36, 405

Searle L, Wilkinson A. \& Bagnuolo W, 1980, Ap. J. 239, 803

Terndrup D.M, Rich R.M. \& Whitford A.E, 1984, PASP, 96, 94

Tinsley B.M, 1972, Ap. J. 178, 319

Tinsley B.M. \& Gunn J.E, 1976, Ap. J. 206, 525

Twarog B.A, 1980, Ap. J. 242, 242

VandenBerg D, 1985, Ap. J. Suppl, 58, 711

de Vaucouleurs G, 1961, Ap. J. Suppl, 5, 223

Visvanathan N. \& Sandage A.R, 1977, Ap. J. 216, 214

Williams T.B, 1976, Ap. J. 209, 716

Whitford A.E, 1977, Ap. J. 211, 527

1978, Ap. J. 226, 777

1985a, PASP, 97, 205

1985b, in Spectral Evolution of Galaxies, C. Chiosi \& A.

Renzini, eds. (Dordrecht, Reidel), P157

Whitford A.E \& Rich R.M (WR), 1983, Ap. J. 274, 723

Wood D.B, 1966, Ap. J. 145, 36

Wood P.R. \& Bessell M.S, 1983, Ap. J. 265, 748

Wu C-C, Faber S.M, Gallagher J.S, Peck M. \& Tinsley B.M, 1980, Ap. J. 237, 290

\section{DISCUSSION}

FREEMAN: At the ST meeting last week, Demarque mentioned new work on evolution of stars more metal-rich than solar. This suggested that the incidence of evolved uv bright stars may be higher in very metal-rich systems. Would this help significantly in reducing the need for younger populations in ellipticals?

PICKLES: The observations do not reveal large numbers of hot evolved stars. Rose's observations in particular set strong limits on the numbers of $A$ or $B$ spectral type stars, so $I$ would be surprised if they were shown to be expected in large numbers in metal-rich populations.

FREEMAN: i) Do you include red horizontal branch stars in your intermediate abundance components, and $i$ il do you think that a smoothly changing abundance could explain the constancy of $\mathrm{HB}$ with $\mathrm{Mgz}$ shown earlier by George Efstathiou?

BURSTEIN: I think George Efstathiou's data may present a serious problem to 'closed system' models: George's data suggest that the $\mathrm{Mgz}-\mathrm{Fe}$ gradient within a galaxy is different from the $M g=-F e$ correlation between galaxies. If this difference is substantiated by future data, then the center of an elliptical is anything but a closed system.

PICKLES: i) The horizontal branch components are not extensive and include only metal-weak standards. ii) One possible explanation for the 
observed radial line strength varlations is that stellar ages might increase radially outwards in ellipticals. The table shows (top rows) HI $(H \alpha+H \beta)$, (middle rows) Mgb, and (lower rows) $F e+C N$ equivalent widths in angstroms, measured in a variety of synthetic spectra constructed to match selected Yale isochrones. There are problems with these synthetic spectra, but the trends in line strength should be correct.

\begin{tabular}{|c|c|c|c|c|}
\hline$\left\langle\mathrm{Fe}_{\mathrm{\theta}} \mathrm{H}\right\rangle$ । Age in Gyr & $\underline{5}$ & $\underline{10}$ & $\underline{15}$ & \\
\hline \multirow[t]{3}{*}{0.4} & 4 & 4 & 3 & $(H \alpha+H \beta)$ \\
\hline & 22 & 24 & 26 & $(M g b)$ \\
\hline & 14 & $\mathrm{n}^{2} \mathrm{a} d$ & 16 & $(\mathrm{Fe}+\mathrm{CN})$ \\
\hline \multirow[t]{3}{*}{0.0} & 5 & $a \geq 4$ & 4 & \\
\hline & 17 & 21 & 24 & \\
\hline & 9 & 11 & 12 & \\
\hline \multirow{3}{*}{-0.6} & 7 & $\begin{array}{l}a \\
r\end{array}$ & 4 & \\
\hline & 8 & 13 & 16 & \\
\hline & 2 & 5 & 7 & \\
\hline
\end{tabular}

\begin{abstract}
Observations show Mgb strength decreasing and $H \beta$ increasing with decreasing luminosity in elliptical nuclei. This implies that metallicity decreases with luminosity and favours young ages in the nuclei, so E nuclei may fall along the vertical line in the table. Mgb strength also decreases radially, whereas $H B$ stays constant. This would be the case if the mean stellar age increased outwards in addition to the mean metallicity declining: ie. the diagonal line in the table. The $\mathrm{Fe}+\mathrm{CN}$ index closely parallels trends in $\mathrm{Mg}$ strength and it is not obvious what population variations would break this correspondence. Dwarf enrichment could give strong $\mathrm{Mgb}$ and $\mathrm{NaD}$ features, and mixing processes may give enhanced $\mathrm{CN}$.
\end{abstract}

CANIZARES: It may be that winds have cleared out the gas in low mass galaxies, but we know that this cannot be true in high mass galaxies because we see their X-ray emission. Doesn't this make you suspicious about the validity of the particular scenario of your synthesis models?

PICKLES: We see hot, metal-enriched gas surrounding (particularly) the dominant galaxies in clusters. Arimoto and Yoshii's models offer a plausible early origin for this gas although they have not treated its subsequent progress through the cluster or cooling and infall. I don't see how purely low mass star formation would generate the observed metallicities in giant ellipticals. This needs supernovae which will expel residual gas from a "spherical system when the thermal energy is high enough.

MOULD: (Comment) The need for supernova driven winds stems from the need to reproduce the mass-metallicity relation in elliptical galaxies. Without the wind to switch of $f$ the chemical evolution in low mass systems, we could not easily produce dwarfs that are metal poor. 